\title{
Gabapentin Targeting and Bone Mineralization Defects: Proposed mechanism for increased fracture risk in patients taking GBP-class anti- epileptic drugs
}

\author{
Jonathan A. Wheeler ${ }^{1}$, Megan L. Noonan², William R. Thompson ${ }^{3}$, Kenneth E. \\ White ${ }^{2}$ \\ ${ }^{1}$ Indiana University School of Medicine, ${ }^{2}$ Department of Medical and Molecular \\ Genetics, Indiana University School of Medicine, ${ }^{3}$ Department of Physical \\ Therapy, Indiana University School of Health and Human Sciences
}

\section{Background and Hypothesis:}

Gabapentin (GBP) is an anti-epileptic drug and first-line therapy for neuropathic pain prescribed to 43 million patients in the US. Unfortunately, GBP use is associated with metabolic bone disease, leading to a 2 to 6 -fold increased fracture incidence. Until now, the pathophysiology of this drug-induced bone loss was unknown. We hypothesize that the impaired bone mineralization and skeletal defects is a result of downstream effects of GBP targeting of the $\alpha_{2} \delta_{1}$ subunit, the only known GBP receptor.

\section{Project Methods:}

In vitro: Murine mesenchymal progenitor cells (MPC-2) were treated with GBP doses varying from $0.5 \mathrm{mM}$ to $50 \mathrm{mM}$ while undergoing osteoblast differentiation for 1 or 2 weeks. Mineralization was assessed by Alizarin red stain. Gene expression was measured by RT-qPCR.

In vivo: The bone phenotype of mice lacking the $\alpha_{2} \delta_{1}$ subunit was analyzed by longitudinal DXA analyses and examined histologically.

\section{Results:}

In vitro: MPC-2 cells treated with 50mM GBP while differentiating for 1 and 2 weeks had decreased osteoblast mineralization and a 7 -fold reduction and 4 fold reduction, respectively, in DMP1.

In vivo: Male and female $\alpha_{2} \delta_{1}$ knockout mice showed a significant decrease in whole body longitudinal ( $6 \mathrm{wk}-18 \mathrm{wk}$ ) bone mineral density (BMD) in males $(p<0.001)$ and females $(p=0.014)$, along with severe osteomalacia, displaying unmineralized osteoid in the trabecular compartment of the distal femoral metaphyses.

\section{Conclusion and Potential Impact:}

The impaired mineralization observed following $\alpha_{2} \delta_{1}$ deletion, coupled with reduced differentiation of MPC-2 cells treated with GBP suggests that GBP regulates bone quality through a novel mechanism influencing phosphate wasting or 1,25 vitamin $\mathrm{D}$ deficiency leading to fracture. With this awareness physicians can monitor these patients for bone mass loss and prescribe drugs to prevent AED-mediated fracture. 